\title{
THE IMPORTANCE OF STAPHYLOCOCCI IN INFANTILE PARENTERAL GASTRO-ENTERITIS
}

\author{
BY \\ PAOLO TOLENTINO \\ From the Department of Paediatrics, University of Genoa
}

(RECEIVED FOR PUBLICATION SEPTEMBER 6, 1951)

The acute nutritional diseases of infants (dyspepsia, gastro-enteritis, enterocolitis, toxicosis) comprise a considerable proportion of infantile diseases, representing nearly $10 \%$ of our hospital cases (1940-1950). There was a fall in the number of cases following the peak year 1945 but there has been a steady increase since (Fig. 1).

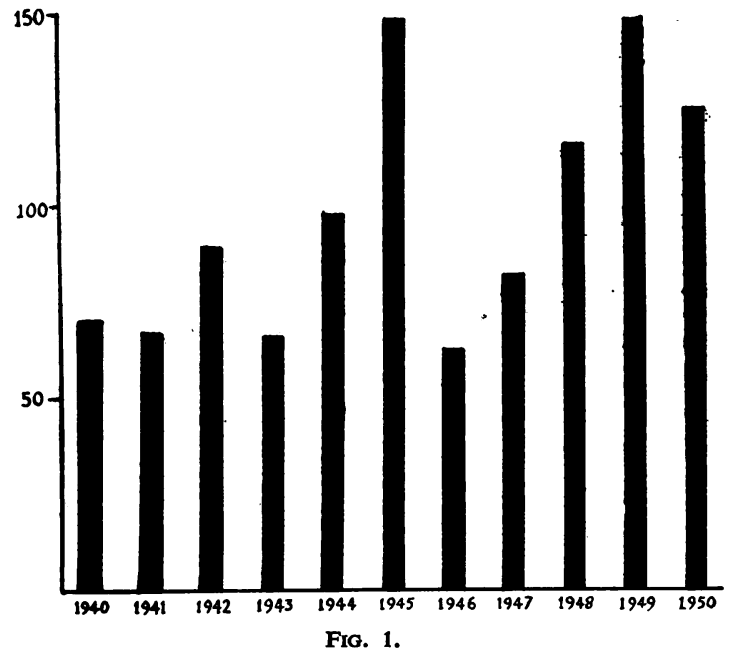

Various reasons lead us to believe that the increase during the last few years has been due not to alimentary or primary enteritic forms, but in the main to infectious parenteral forms: (1) the extreme rarity of isolating shigellae or salmonellae from the stools (Tolentino, 1950); (2) the absence of positive agglutinations for shigellae in healthy subjects (Tolentino, 1951, unpublished data), in contrast to the frequency of such agglutinations in healthy subjects in the endemic shigellosis areas (D'Alessandro and Burgio, 1945); (3) variations noted in the seasonal incidence of the disease which showed a peak during the summer of 1945 (Fig. 2) but, in contrast, an irregular curve, less inclined to rise during the summer and remarkably intensified during the winter of 1950 (Fig. 3).
The modern prevalence of enteric forms of parenteral origin also appears in other parts of the

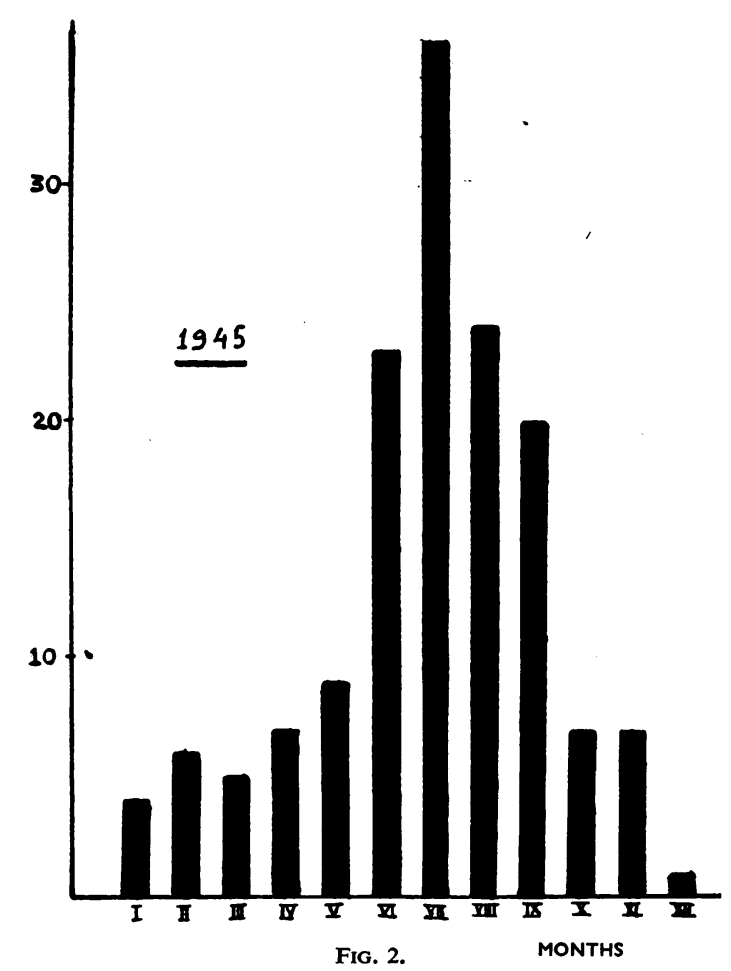

world (Hurtado and Aballi, 1949), and appear to be an attribute of the more civilized countries, where hygienic and economic conditions are better (e.g. water supply, use of powdered milk), whereas elsewhere there is a prevalence of primary enteritic disease.

In the parenteral forms much attention has been paid to the possibility of a virus aetiology (Light and Hodes, 1949, and others), and indeed, some epidemic forms seem to be almost certainly of this 
nature. The fact must not be overlooked, however, that even serious enteritic infections may be caused by pyogenic bacteria, notably staphylococci, and the latter have been the subject of our interest for several years.

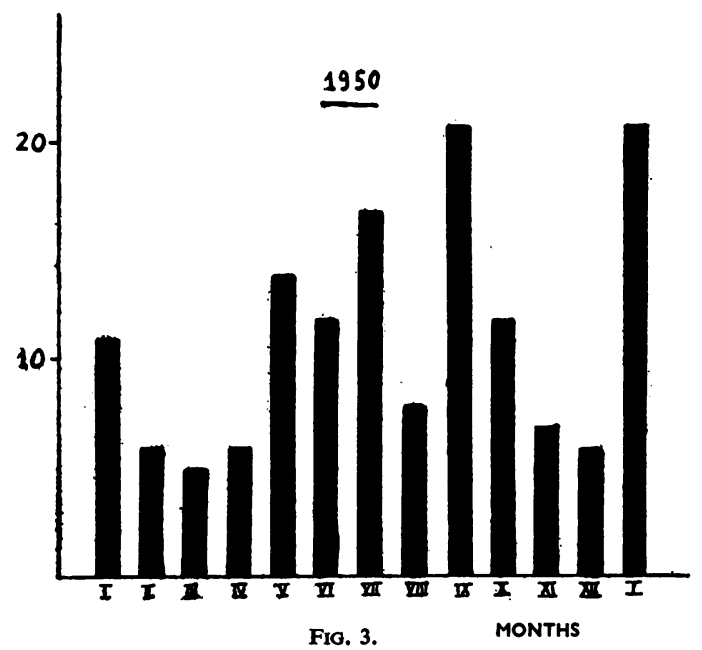

The various ways in which gastro-enteritis may develop from an infective parenteral focus may be summarized as (1) decrease of intestinal secretions (in connexion with the disease) and consequent stagnation of the intestinal contents, the ascent of bacteria towards the small intestine, abnormal formations of amines, of organic acids and of bacterial and alimentary toxins, with irritation of the mucous membrane and subsequent hyperperistalsis or antiperistalsis; (2) elimination of toxins from parenteral infective foci through the intestine, and action developed by these toxins on the intestinal mucosa and nerve plexuses; (3) toxic action of bacterial products elaborated in the parenteral foci on the central nervous system, and on the neuro-vegetative centres, with reflex-induced diarrhoea and vomiting.

The first of these mechanisms is authoritatively upheld by American workers (Nelson, 1950). We have been able to demonstrate the truth of the second mechanism by showing the elimination of staphylococcal haemolytic toxins by the intestine and their presence in the stools of children suffering from staphylococcal parenteral infections (pharyngitis, otitis, impetigo) accompanied by diarrhoea (Tolentino, Ravasi and Durand, 1949). The third mechanism, which had been suggested by French workers (Lévesque, Bastin and Lafourcade, 1949), has seemed to us to be worthy of attention, since we have noticed that the presence of adsorbent foods in the intestine does not always prevent diarrhoea, as should be the case if the only mechanism were that of the irritative action of the toxins present in the intestinal lumen (Tolentino, 1949). We have thus reached the hypothesis that some enteritic forms may be caused by enterotoxic staphylococci with a mechanism acting on the central nervous system. We have carried out an investigation on sucklings suffering from gastro-enteritis with pharyngitis by isolating the pharyngeal staphylococci on Buttiaux and Brogniart's (1947) culture media, and by testing the enterotoxic activity of a filtrate of the culture from Dolman and Wilson's (1938) medium in kittens. The first positive case was reported by Tolentino and Ravasi in 1950. Four similar cases have since been studied (Tolentino and Ravasi, 1951). We have found enterotoxic staphylococci in the pharynx of $2 \%$ of healthy children and of $26 \%$ of children suffering from gastro-enteritis with pharyngitis. Non-enterotoxic staphylococci were isolated in $50 \%$ of healthy children and in $37 \%$ of infants with gastro-enteritis (Fig. 4).

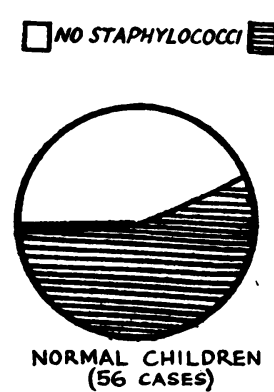

(56 CASES)

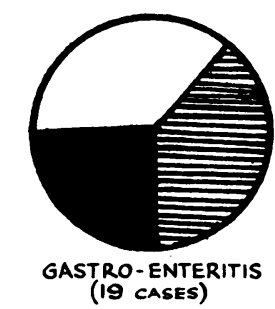

FIG. 4.
In those cases of gastro-enteritis with pharyngitis, where the staphylococcus is not found, the cause may be different, presumably being due to a virus, but it is interesting that in as many as $26 \%$ of our cases the staphylococcus was responsible for both pharyngitic and gastro-enteritic symptoms. The suckling appears to be more sensitive than the adult to the action of the enterotoxin, since for enteritic symptoms to be produced in the latter it seems necessary for them to have eaten food contaminated by a carrier of infection (Wilson, 1938). It must also be noted that, unlike the strains isolated from contaminated food, the strains isolated from children's pharynxes lose their enterotoxin-producing capacity in a few weeks: 
it seems, therefore, that this feature is less consistently connected with their type.

The mode of action of the enterotoxin has been shown by the administration of the toxin in large doses to animals (Tolentino and Bellieni, 1951). These workers produced lesions of the central nervous system, diapedetic haemorrhages (Fig. 5) and degenerate lesions of the nerve centres (Fig. 6) namely, lysis of the tigroid, pyknosis of the nuclei, and pseudo-neuronophagia. On injecting large doses of the toxin, the animal, instead of showing the classical symptomatology of diarrhoea and vomiting, merely exhibited a neurological symptomatology characterized by convulsions, opisthotonus, ataxia, hallucinations, and coma and death within 24 hours. It therefore appears possible that enterotoxin acts through a neurotoxic mechanism and that both vomiting and diarrhoea may also be originated by a central mechanism. Not only does such an assumption imply a central nervous origin for these cases of gastro-enteritic symptomatology, but it also supports the suggestion that even some cases of toxicosis, such as the

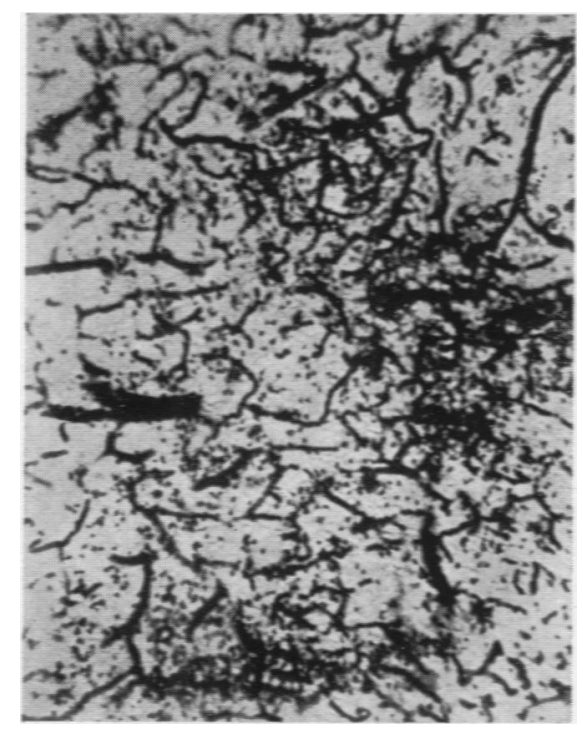

Fig. 5.-Photomicrograph showing diapedetic hacmorrhages in the central nervous system in experimental animals (Pickworth's method $\times 55$ ). encephalitic type with scanty gastro-enteritic signs, may have a staphylococcal origin.

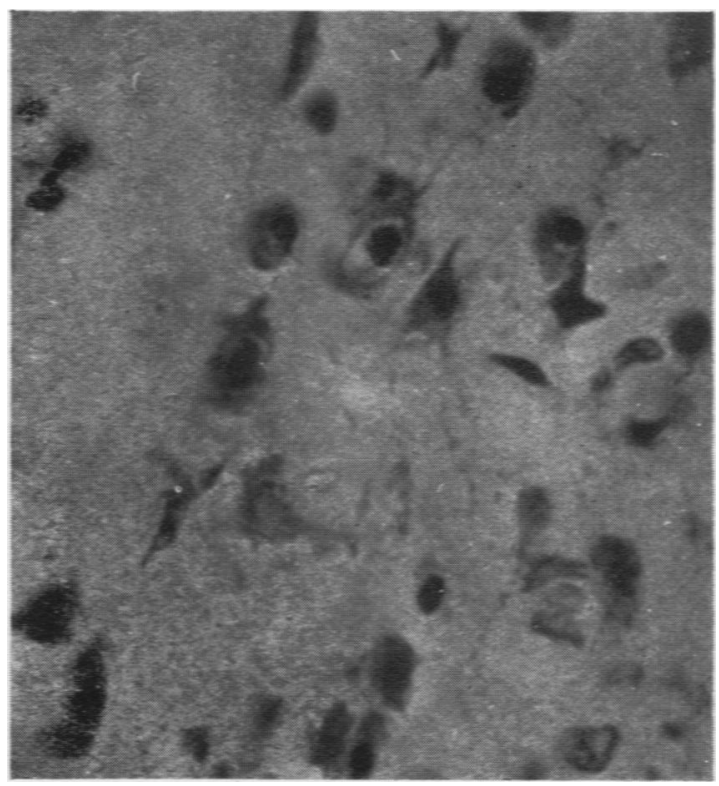

Fig. 6.-Photomicrograph showing degenerate lesions of the nerve centres $(\mathrm{Nis} d \times 600)$.

\section{REFERENCES}

Buttiaux, R. and Brogniart, R. (1947). Ann. Inst. Pasteur, 73, 830.

D'Alessandro, G. and Burgio, G. R. (1945). G. Med., 2. fasc. 2-3.

Dolman, C. E. and Wilson, R. J. (1938). J. Immunol., 35, 13.

Hurtado, F. and Aballi, A. J. (1949). 2nd Congr. Panam. Ped. Mexico.

Lévesque, J., Bastin, R. and Lafourcade, J. (1949). $X I{ }^{C}$ Congr. Ped. Langue Franc. Paris.

Light, J. S. and Hodes, H. L. (1949). J. exp. Med., 90, 113.

Nelson, W. (1950). In Mitchel-Nelson Textbook of Pediatrics. Philadelphia.

Tolentino, P. Unpublished data.

(1949). XX Congr. Ital. Ped. Taormina. (1950). Amn. paediat. (Basel), 175, 201.

and Bellieni, G. (1951). Pediatria (Napoli), 59, 309.

- and Ravasi, R. M. (1950). Minerva pediat. (Torino), 2, 172,

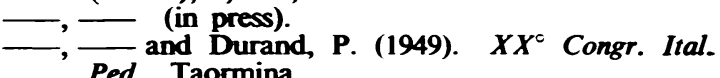
Ped. Taormina.

Wilson, R. J. (1938). Canad. publ. Hlth. J., 29, 329. 